\title{
Construction of tissue microarrays from prostate needle biopsy specimens
}

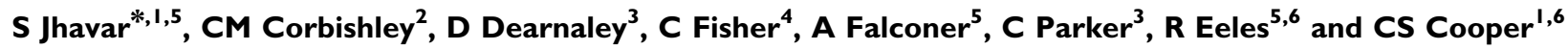 \\ 'Sections of Molecular Carcinogenesis, Institute of Cancer Research, Sutton, Surrey SM2 5NG, UK; '²Department of Cellular Pathology, St George's \\ Healthcare Trust, Blackshaw Road, Tooting, London SWI 7 OQT, UK; ${ }^{3}$ Department of Academic Urology, Royal Marsden NHS Foundation Trust and \\ Institute of Cancer Research, Sutton, Surrey SM2 5PT, UK; ${ }^{4}$ Department of Histopathology, Royal Marsden NHS Foundation Trust, Fulham Road, London, \\ SW3 6Jj, UK; ${ }^{5}$ Section of Cancer Genetics, Institute of Cancer Research, Sutton, Surrey SM2 5NG, UK
}

Needle biopsies are taken as standard diagnostic specimens for many cancers, but no technique exists for the high-throughput analysis of multiple individual immunohistochemical (IHC) markers using these samples. Here we present a simple and highly reliable technique for constructing tissue microarrays (TMAs) from prostatic needle biopsies. Serial sectioning of the TMAs, called 'Checkerboard TMAs', facilitated expression analysis of multiple proteins using $\mathrm{HC}$ markers. In total, 100\% of the analysed biopsies within the TMA both preserved their antigenicity and maintained their morphology. Checkerboard TMAs will allow the use of needle biopsies (i) alongside other tissue specimens (trans-urethral resection of prostates and prostatectomies in the case of prostate cancer) in clinical correlation studies when searching for new prognostic markers, and (ii) in a diagnostic context for assessing expression of multiple proteins in cancers from patients prior to treatment.

British Journal of Cancer (2005) 93, 478-482. doi:I0.I038/sj.bjc.6602726 www.bjcancer.com

Published online 9 August 2005

(C) 2005 Cancer Research UK

Keywords: prostate; cancer; needle biopsy; tissue microarray; immunohistochemistry

Needle biopsy specimens are taken at the time of diagnosis from many human malignancies, including oral cancer, breast cancer, sarcomas, prostate cancer and lymph node masses (Bostwick, 1997; Morrow et al, 2002; Neville and Day, 2002; Cormier and Pollock, 2004; Jereczek-Fossa et al, 2004). In prostate cancer, for example, examination of needle biopsy specimens yields valuable information, including Gleason grade and the tumour extent, that facilitates decision-making on the appropriate treatment. A major current problem in prostate cancer is to predict the behaviour of early and potentially localised disease (Yao and Lu-Yao, 2002). Some cases may remain dormant for many years without progressing, while others will progress rapidly to metastases. It is therefore important to identify the patients who need to be treated and separate them from those who can be managed by active surveillance, thus sparing the latter of the adverse consequences of unnecessary treatment (Dearnaley and Melia, 1997; Parker, 2004). If tests are to be developed that will allow the prediction of clinical behaviour of patients diagnosed with prostate cancer, for example, following prostate-specific antigen (PSA) screening, it must be possible to perform the test on biological specimens obtained from the patients at the time of diagnosis,

\footnotetext{
*Correspondence: Dr S Jhavar, Section of Molecular Carcinogenesis, Male Urological Cancer Research Centre, Institute of Cancer Research, I 5 Cotswold Road, Sutton, Surrey SM2 5NG, UK;

E-mail: sameerj@icr.ac.uk

${ }^{6}$ Joint senior authors.

Received 28 February 2005; revised 8 June 2005; accepted 20 June 2005; published online 9 August 2005
}

which would usually only include trans-rectal ultrasound (TRUS)guided needle biopsy specimens, blood or urine.

Tissue microarrays (TMAs) that allow many hundreds of tumours to be analysed simply in a single experiment have proven to be very useful in high-throughput evaluation of prognostic markers for predicting the clinical behaviour of patients with prostate cancer (Dhanasekaran et al, 2001; Rhodes et al, 2003; Foster et al, 2004). At the moment, construction of TMAs in prostate cancer studies is however limited to tissue samples obtained from punched-out cores from paraffin blocks from transurethral resection of prostate (TURP) specimens and from radical prostatectomies. Battifora and Mehta (1990) described a Checkerboard method for producing tissue arrays as a forerunner to the core-based TMA method described by Kononen et al (1998). In this Checkerboard method, multiple (up to 100) chunks of formalin-fixed de-paraffinised or fresh normal or tumour tissue were reset in agar and then in paraffin wax in a Checkerboard pattern. This procedure is unsuitable for formalin-fixed needle biopsy specimens because, following dewaxing, the tiny specimens are easily lost and very difficult to orientate and re-embed vertically. The TMA procedure described by Kononen et al (1998) is also unsuitable for use with prostate needle biopsy specimens. The diameter of these needle biopsies, which are conventionally embedded horizontally in paraffin blocks, is less than $1.26 \mathrm{~mm}$, the outer diameter of an $18-\mathrm{G}$ needle, and their depth is further eroded after the block has been sectioned for diagnosis. Hence, cores obtained by the Kononen et al (1998) method would be extremely difficult to align in the recipient block, and at best would provide only a few slices. We present an approach for constructing TMAs from needle biopsy specimens that overcomes these difficulties. 


\section{MATERIALS AND METHODS}

\section{Selection of paraffin blocks containing prostate needle biopsies}

Paraffin-embedded formalin-fixed prostate needle biopsy specimens (Figure 1A) were obtained from prostate cancer patients (i) who took part in the RT01 trial at The Royal Marsden Hospital NHS Trust and had given written consent for research to be undertaken on their biopsies (including their 2-year post radiotherapy biopsies) $(n=2)$; (ii) who had diagnostic TRUS-guided prostate biopsies at the St Georges Hospital NHS Trust $(n=23)$. The biopsies from the former group of patients were only used in the pilot experiments (data not shown). Biopsies from the latter group of patients were used in the validation set of TMAs and all patients in this group had died at the time of the study.

\section{Antibodies}

The following antibodies were chosen: (i) low-molecular-weight keratin, CAM 5.2 (cat\# 345779, supplied by Becton Dickinson, dilution 1:5), chosen to show all epithelial cells; (ii) highmolecular-weight keratin, LP34 (cat\# M0717, supplied by DakoCytomation, dilution 1:50) that stains only basal cells and (iii) PSA (rabbit polyclonal, cat\# A0562, supplied by DakoCytomation, dilution $1: 20000)$ that stains prostatic epithelial cells of acini and peripheral ducts.

\section{Immunohistochemistry (IHC)}

Sections of the Checkerboard TMA were cut at $3 \mu \mathrm{m}$ onto Superfrost Plus ${ }^{\mathrm{TM}}$ positively charged glass slides (Menzel-Glaser, Germany), dewaxed with xylene and rehydrated to water through graded ethanol rinses. Endogenous peroxidase activity was blocked by immersion of tissue sections in a $10 \%\left(\mathrm{vv}^{-1}\right)$ solution of $\mathrm{H}_{2} \mathrm{O}_{2}$ in deionised water at room temperature for $8 \mathrm{~min}$. In all, 11 of diluted (1:10 in deionised water) Tris-EDTA-citrate ( $\mathrm{pH} 7.8)$ buffer was preheated in a pressure cooker (Nordicware microwave tender cooker, Bio Genex) for 16 min without the slides. Hightemperature antigen retrieval was then performed by placing the peroxidase-blocked slides in the hot buffer and sealing the pressure cooker, and comprised microwave (Panasonic 900 W) heating at high temperature for $6 \mathrm{~min}$. After this, the pressure was released and cold water was allowed to run in the pressure cooker to cool the slides, which were now ready for staining. Chymo- trypsin pretreatment was performed by placing the peroxidaseblocked slides in a chymotrypsin (Alpha Chymotrypsin Type II bovine pancreas, Sigma Cat\# C-4129) solution in Tris buffer ( $\mathrm{pH}$ 7.6) for $30 \mathrm{~min}$. The slides were then washed by running tap water on them for $5 \mathrm{~min}$. Staining after the primary antibody incubation involved the use of ChemMate Envision Kit K5007 from Dakocytomation, and the Optimax Plus Staining Machine (BioGenex, supplied by A Menarini) and manufacturer's instructions were followed. Sections were incubated with the primary antibody for $30 \mathrm{~min}$ at room temperature and washed with buffer (Optimax Wash buffer - BioGenex supplied by A Menarini Cat. No. HK583$5 \mathrm{~K})$.

\section{RESULTS}

\section{Preparation of individual blocks or 'checkers'}

The starting point for construction of Checkerboard TMAs was paraffin donor blocks containing formalin-fixed prostate needle biopsy specimens embedded at their surface (Figure 1A). A scalpel blade was used to cut $4 \mathrm{~mm}$ lengths of biopsy specimens corresponding to areas of tumour or normal tissue identified from the original haematoxylin and eosin (H\&E)-stained longitudinal section by the study histopathologist (CMC). Additional cuts were made in the wax to obtain a $4 \times 2 \times 2 \mathrm{~mm}^{3}$ cubical shaped block, which we called a 'biopsy checker', containing the $4 \mathrm{~mm}$ length of biopsy specimen attached on one of its $4 \times 2 \mathrm{~mm}^{2}$ faces. To help orientate the block in later stages of the procedure, the $4 \times 2 \mathrm{~mm}^{2}$ face opposite that containing the biopsy specimen was immediately painted with dyes - blue and red dyes were used to paint this face on alternate checkers (Figure 1B).

\section{Construction of the Checkerboard TMA}

The checkers were then arranged in a square grid pattern in a $36 \times 24 \times 5 \mathrm{~mm}^{3}$ stainless steel mould (Raymond A Lamb Ltd, UK) (Figure 1C). The checkers were positioned in the mould so that each biopsy specimen had a vertical orientation allowing analysis of the cross-section of each biopsy. The mould was placed on the semiheated platform of the embedding apparatus, while the checkers were arranged. A predetermined template was used as a guide to position and record the identity of each checker within the array. A plastic cassette was then placed over the mould. The checkers were then re-embedded by pouring hot paraffin wax $\left(60^{\circ} \mathrm{C}\right)$ into the mould through the cassette and the mould was then cooled by placing it on a cold platform $\left(-5^{\circ} \mathrm{C}\right)$ for $1 \mathrm{~h}$. Gentle
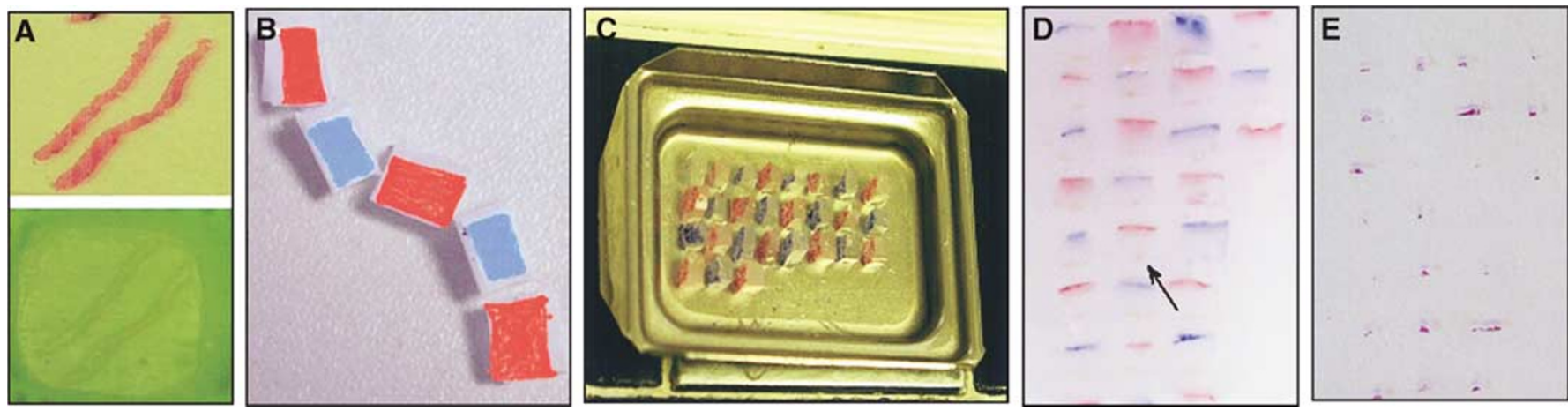

Figure I Construction of Checkerboard TMA from prostatic needle biopsies. (A) Prostatic needle biopsies embedded horizontally within a paraffin block (bottom) and H\&E sections from this block (top). (B) Checkers $\left(4 \times 2 \times 2 \mathrm{~mm}^{3}\right)$ cut from the original paraffin block containing the prostatic needle biopsy specimen was painted on the surface opposite that containing the biopsy, alternately with blue and red dyes. (C) Checkers positioned in a $36 \times 24 \times 5$ mm ${ }^{3}$ stainless steel mould (Raymond A Lamb Ltd, UK) so that each biopsy specimen now had a vertical orientation. (D) Finished Checkerboard TMA block in which the cross-sections of the vertically positioned biopsies are seen (arrow). (E) Haematoxylin and eosin-stained section (4 $\mu \mathrm{m})$ obtained from the Checkerboard TMA block. 
rocking of the mould in a circular fashion was carried out while the hot paraffin wax was being poured to ensure that each checker was completely surrounded by wax. Sufficient care was taken to avoid formation of air bubbles in the wax between the checkers. The dyed surfaces of the checkers are monitored: (i) to mark the position of the checkers within the finished paraffin block attached to the plastic cassette and (ii) to ensure that the checkers remain in their correct orientation during the setting process. The alternate marking of individual checkers with blue and red dyes gave rise to a Checkerboard appearance (Figure 1D). Initial sections $(4 \mu \mathrm{m})$ cut onto Superfrost ${ }^{\mathrm{TM}}$ glass slides (Ultima) were stained by conventional H\&E stains to identify the location and morphology of representative biopsy specimens within the microarray block (Figure 1E).

\section{Analysis of IHC markers using Checkerboard TMAs}

Tissue microarrays were constructed from biopsies from deceased prostate cancer patients $(n=23)$. In all, 45 checkers were prepared from needle biopsies from these 23 patients. Out of the 45 checkers, 41 retained their biopsy specimens in the Checkerboard TMA, equivalent to a tissue loss of $9 \%$. The remaining checkers included 22 biopsy specimens that had regions considered to contain cancer based on the original H\&E section of the horizontally embedded biopsy, and 19 that had been judged as normal.

Results of IHC studies on 123 individual tissue slices from the Checkerboard TMA demonstrated that antigenicity was preserved in $100 \%$ of the retained biopsies. Results from serial sections
A

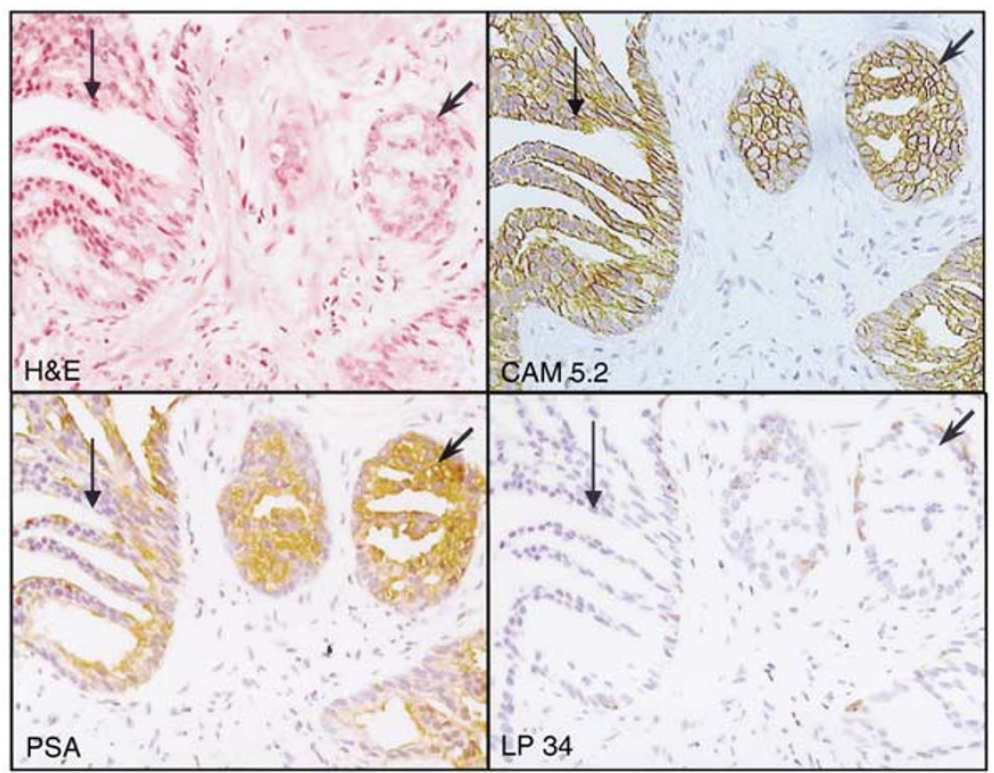

B

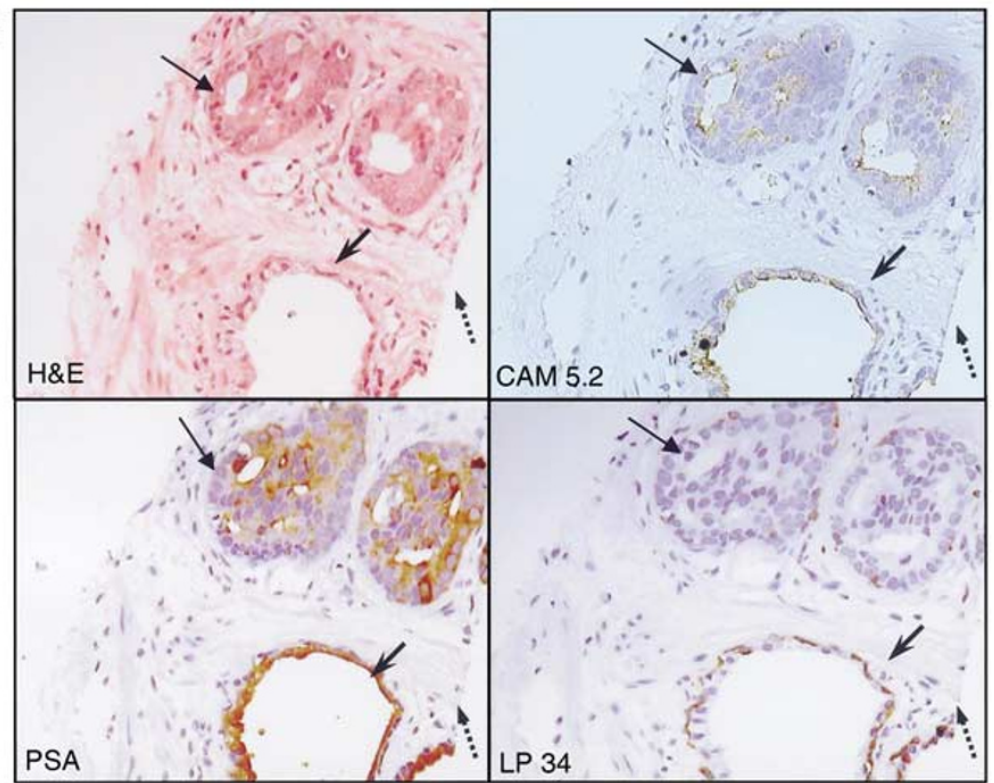

C

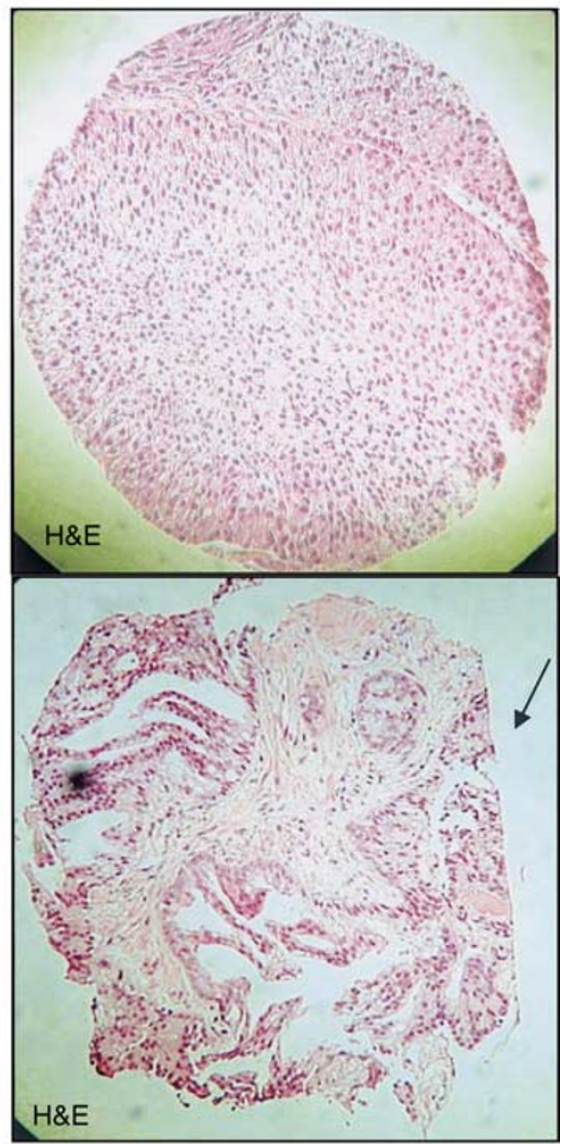

Figure 2 Analysis of sections obtained from a Checkerboard TMA. $(\mathbf{A})$ Serial sections $(\times 20)$ from a single biopsy specimen that contained both cancer (thin arrow) and prostatic intra-epithelial neoplasia (PIN) (arrowhead) stained by H\&E, PSA, CAM 5.2 and LP34. (B) Serial sections $(\times 20)$ of second biopsy specimen containing both cancer (thin arrow) in the deeper part of the biopsy and benign glands on the surface (arrowhead) stained by H\&E, PSA, CAM 5.2 and LP3. The surface of the biopsy that was originally cut for diagnosis of cancer is seen (dotted arrow). $(\mathbf{C})$ Comparison of a tissue core $(\times 20)$ from conventional TMA (top panel) with a biopsy core $(\times 20)$ from the Checkerboard TMA (lower panel). The side of the needle biopsy originally cut for diagnostic purposes can be seen (arrow). 
$(3-4 \mu \mathrm{m})$ of two representative biopsy specimens within the Checkerboard TMA containing cancer stained by H\&E, PSA, CAM 5.2 (low-molecular-weight keratin) and LP34 (high-molecularweight keratin) are shown (Figures 2A and B). The PSA, CAM 5.2 and LP34 antibodies are used routinely in the diagnosis of prostate cancer (Moll et al, 1982; Makin et al, 1984; Gillespie et al, 2002; Evans, 2003). An interesting feature of the Checkerboard TMA analyses was that cancer was identified in nine out of 19 (five out of 19 based on H\&E staining alone) checkers containing biopsy specimens originally judged as normal.

A comparison of a section of core obtained from conventional TMA with that obtained from Checkerboard TMA method described in this report is shown in Figure 2C. The cross-sections are very similar in size, but the tissue specimen from the Checkerboard TMAs generally has a more irregular shape and the side of the needle biopsy originally cut for diagnostic purposes can usually be seen (Figure 2C, arrow).

\section{DISCUSSION}

We have demonstrated that it is possible to construct TMA from diagnostic prostate needle biopsies. Prostate needle biopsies are formalin fixed and set horizontally on the surface of a paraffin wax block so that a lengthwise slice of the entire biopsy specimen can be taken for diagnostic examination. Our method for constructing TMAs from these specimens involves cutting out small $4 \mathrm{~mm}$ lengths of the biopsy specimen and repositioning them in a vertical orientation so that approximately $90-1004-\mu \mathrm{m}$-thick sections from each biopsy can be made available for IHC staining, in comparison to only a few from the original horizontally embedded specimens. Such formalin-fixed material could also, in principle, be used for other molecular procedures such as fluorescence in situ hybridisation (FISH) (Takahashi et al, 1994; Bastacky et al, 2004).

The IHC staining results from our study show that the biopsy tissue maintains its antigenicity even after embedding at the surface of the paraffin block and re-embedding the biopsy checkers in the Checkerboard TMAs. Using conventional TMA technology, Rubin et al (2002) concluded that less than three cores may not accurately represent protein expression, but that more than four cores were unnecessary and did not add to the value of arrays used to predict prostate cancer outcome. In the method described here, multiple assessments of protein expression could be made by analysing tumour from different biopsy cores or from tumour at different depths within an individual core. Notably, the $\mathrm{H} \& \mathrm{E}$ and IHC analyses of cross-sections of cores in the Checkerboard TMAs detected cancer in nearly one-half of biopsies originally judged as normal by conventional H\&E analyses of the biopsies. Such a finding may in part be due to the improved sensitivity allowed by use of H\&E and IHC in combination, and in part achieved by the inherent advantage of this technique in being able to examine a greater depth of tissue. In the current series, this finding did not result in any new cancer diagnosis because all samples were taken from patients who had already been diagnosed with prostate cancer. However, in future studies, it may well be interesting to compare the results obtained in conventional diagnosis using needle biopsy specimens with those obtained from H\&E and IHC analyses on cross-sections from Checkerboard TMAs.

Other investigators have reported a $10-30 \%$ loss of tissue when constructing conventional TMAs from a large number of cores using manual or automated methods (Schraml et al, 1999; Mucci et al, 2000; Hoos and Cordon-Cardo, 2001; Mengel et al, 2003). This compares to a $9 \%$ tissue loss reported in this study. Our low level of loss may indicate that re-embedding the checkers using hot paraffin wax holds the cores more tightly compared to conventional TMA, where the recipient wax block is softened at $36^{\circ} \mathrm{C}$ to secure the cores.

Needle biopsies provide vital information about the biology and natural history of the cancer (Sotiriou et al, 2002) and microarray studies are showing great promise in providing diagnostic as well as prognostic information in a variety of cancers (Pusztai et al, 2003). Tissue microarray studies in prostate cancer evaluating predictive markers have, however, been limited to TURP and radical prostatectomy specimens (Bubendorf et al, 1999; Dhanasekaran et al, 2001; Foster et al, 2004). Trans-rectal ultrasoundguided biopsies now predominantly provide diagnostic tissue, but are in general unsuitable for construction of TMA using conventional methods, because of the orientation of the small biopsies. The technique for the production of TMAs from needle biopsy specimens allows formalin-fixed prostatic needle biopsy specimens to be used: (i) in a diagnostic context for assessing multiple IHC markers in patients prior to treatment and (ii) together with both TURP and radical prostatectomy specimens in high-throughput screening for new prognostic markers. The analysis of multiple markers in prostate cancer is important because it may be the analysis of a combination of markers rather than a single marker that provides the best prognostic information (Tricoli et al, 2004). This technique may additionally be applicable in analysing needle biopsies taken from other cancer types.

\section{ACKNOWLEDGEMENTS}

We are grateful to Sue Clinton for her initial help in cutting the TMA blocks, Dr Nasiri for his help in initial reviewing of the biopsy slides, David Hudson and Sian Rizzo for their help in taking photomicrographs of the TMA sections and Anne Fletcher for providing a slide of conventional TMA to photograph the section of a conventional TMA core. This study was funded by (i) The National Cancer Research Institute (NCRI), (ii) The Rosetrees Trust and (iii) The Grand Charity of Freemasons. This work was undertaken in The Royal Marsden NHS Trust, which received a proportion of its funding from the NHS Executive; the views expressed in this publication are ours, and not necessarily those of the NHS Executive. This work was supported by the Institute of Cancer Research, the Bob Champion Cancer Trust and Cancer Research UK Section of Radiotherapy CUK grant number C46/ A2131.

\section{REFERENCES}

Bastacky S, Cieply K, Sherer C, Dhir R, Epstein JI (2004) Use of interphase fluorescence in situ hybridisation in prostate needle biopsy specimens with isolated high-grade prostatic intraepithelial neoplasia as a predictor of prostate adenocarcinoma on follow-up biopsy. Hum Pathol 35: 281-289

Battifora H, Mehta P (1990) The checkerboard tissue block. An improved multitissue control block. Lab Invest 63: $722-724$

Bostwick DG (1997) Evaluating prostate needle biopsy: therapeutic and prognostic importance. CA Cancer J Clin 47: 297-319
Bubendorf L, Kononen J, Koivisto P, Schraml P, Moch H, Gasser TC, Willi N, Mihatsch MJ, Sauter G, Kallioniemi OP (1999) Survey of gene amplifications during prostate cancer progression by high-throughout fluorescence in situ hybridisation on tissue microarrays. Cancer Res 59: $803-806$

Cormier JN, Pollock RE (2004) Soft tissue sarcomas. CA Cancer J Clin 54: $94-109$

Dearnaley DP, Melia J (1997) Early prostate cancer - to treat or not to treat? Lancet 349: $892-893$ 
Dhanasekaran SM, Barrette TR, Ghosh D, Shah R, Varambally S, Kurachi K, Pienta KJ, Rubin MA, Chinnaiyan AM (2001) Delineation of prognostic biomarkers in prostate cancer. Nature 412: $822-826$

Evans AJ (2003) Alpha-methylacyl CoA racemase (P504S): overview and potential uses in diagnostic pathology as applied to prostate needle biopsies. J Clin Pathol 56: $892-897$

Foster CS, Falconer A, Dodson AR, Norman AR, Dennis N, Fletcher A, Southgate C, Dowe A, Dearnaley D, Jhavar S, Eeles R, Feber A, Cooper CS (2004) Transcription factor E2F3 over expressed in prostate cancer independently predicts clinical outcome. Oncogene 23: 5871-5879

Gillespie JW, Best CJ, Bichsel VE, Cole KA, Greenhut SF, Hewitt SM, Ahram M, Gathright YB, Merino MJ, Strausberg RL, Epstein JI, Hamilton SR, Gannot G, Baibakova GV, Calvert VS, Flaig MJ, Chuaqui RF, Herring JC, Pfeifer J, Petricoin EF, Linehan WM, Duray PH, Bova GS, Emmert-Buck MR (2002) Evaluation of non-formalin tissue fixation for molecular profiling studies. Am J Pathol 160: 449-457

Hoos A, Cordon-Cardo C (2001) Tissue microarray profiling of cancer specimens and cell lines: opportunities and limitations. Lab Invest 81: $1331-1338$

Jereczek-Fossa BA, Jassem J, Orecchia R (2004) Cervical lymph node metastases of squamous cell carcinoma from an unknown primary. Cancer Treat Rev 30: 153-164

Kononen J, Bubendorf L, Kallioniemi A, Barlund M, Schraml P, Leighton S, Torhorst J, Mihatsch MJ, Sauter G, Kallioniemi OP (1998) Tissue microarrays for high-throughput molecular profiling of tumour specimens. Nat Med 4: 844-847

Makin CA, Bobrow LG, Bodmer WF (1984) Monoclonal antibody to cytokeratin for use in routine histopathology. J Clin Pathol 37: 975-983

Mengel M, Kreipe H, von Wasielewski R (2003) Rapid and large-scale transition of new tumour biomarkers to clinical biopsy material by innovative tissue microarray systems. Appl Immunohistochem Mol Morphol 11: 261 - 268

Moll R, Franke WW, Schiller DL, Geiger B, Krepler R (1982) The catalog of human cytokeratins: patterns of expression in normal epithelia, tumours and cultured cells. Cell 31: $11-24$

Morrow M, Strom EA, Bassett LW, Dershaw DD, Fowble B, Giuliano A Harris JR, O'Malley F, Schnitt SJ, Singletary SE, Winchester DP (2002) Standard for breast conservation therapy in the management of invasive breast carcinoma. CA Cancer J Clin 52: 277-300
Mucci NR, Akdas G, Manely S, Rubin MA (2000) Neuroendocrine expression in metastatic prostate cancer: evaluation of high throughput tissue microarrays to detect heterogeneous protein expression. Hum Pathol 31: $406-414$

Neville BW, Day TA (2002) Oral cancer and precancerous lesions. CA Cancer J Clin 52: 195-215

Parker C (2004) Active surveillance: towards a new paradigm in the management of early prostate cancer. Lancet Oncol 5: 101-106

Pusztai L, Ayers M, Stec J, Clark E, Hess K, Stivers D, Damokosh A, Sneige N, Buchholz TA, Esteva FJ, Arun B, Cristofanilli M, Booser D, Rosales M, Valero V, Adams C, Hortobagyi GN, Symmans WF (2003) Gene expression profiles obtained from fine-needle aspirations of breast cancer reliably identify routine prognostic markers and reveal large-scale molecular differences between estrogen-negative and estrogen-positive tumours. Clin Cancer Res 9: 2406-2415

Rhodes DR, Sanda MG, Otte AP, Chinnaiyan AM, Rubin MA (2003) Multiplex biomarker approach for determining risk of prostate-specific antigen-defined recurrence of prostate cancer. J Natl Cancer Inst 95: $661-668$

Rubin MA, Dunn R, Strawderman M, Pienta KJ (2002) Tissue microarray sampling strategy for prostate cancer biomarker analysis. Am J Surg Pathol 26: $312-319$

Schraml P, Kononen J, Bubendorf L, Moch H, Bissig H, Nocito A, Mihatsch MJ, Kallioniemi OP, Sauter G (1999) Tissue microarrays for gene amplification surveys in many different tumour types. Clin Cancer Res 5 $1966-1975$

Sotiriou C, Khanna C, Jazaeri AA, Petersen D, Liu ET (2002) Core biopsies can be used to distinguish differences in expression profiling by cDNA microarrays. J Mol Diagn 4: 30-36

Takahashi S, Qian J, Brown JA, Alcaraz A, Bostwick DG, Lieber MM, Jenkins RB (1994) Potential markers of prostate cancer aggressiveness detected by fluorescence in situ hybridisation in needle biopsies. Cancer Res 54: $3574-3579$

Tricoli JV, Schoenfeldt M, Conley BA (2004) Detection of prostate cancer and predicting progression: current and future diagnostic markers. Clin Cancer Res 10: $3943-3953$

Yao SL, Lu-Yao G (2002) Understanding and appreciating overdiagnosis in the PSA era. J Natl Cancer Inst 94: 958-960 